Preprint US-FT / 29-96

revised version, October 1996

\title{
Deviations from the superposition model in a Dual Parton Model with formation zone cascade in both projectile and target nuclei
}

\author{
G. Battistoni \\ INFN, Sezione di Milano, I-20133 Milano, Italy \\ C. Forti \\ INFN, Laboratori Nazionali di Frascati,I-00044 Frascati, Italy \\ J. Ranft \\ Departamento de Física de Partículas, \\ Universidade de Santiago de Compostela, \\ E-15706 Santiago de Compostela, Spain \\ S. Roesler \\ Universität Siegen, Fachbereich Physik,D-57068 Siegen, Germany
}

\begin{abstract}
A Dual Parton Model with a formation zone intranuclear cascade in the spectators of the projectile and target nuclei is studied. The hadrons produced in the formation zone cascade contribute to Feynman- $x_{F}$ and lab- $x$ distributions in the fragmentation regions of the target and projectile nuclei. We discuss the consequences of this model in the secondary cosmic ray production, by analyzing the calculated spectrum weighted moments for pion and kaon production. We show that the proposed model leads to significant differences with respect to a simple superposition model, where the nucleus-nucleus collision is replaced by a few corresponding nucleonnucleus collisions.
\end{abstract}




\section{Introduction}

Nucleus-nucleus collisions are of great importance for the understanding of the cosmic ray cascades in the atmosphere. Models for sampling hadron production events in nucleus-nucleus and hadron-nucleus collisions are needed for the simulation of the development of cosmic ray showers in atmosphere. In some models used for the simulation of cosmic ray cascades, examples are HEMAS [1] and SIBYLL [2], the correct treatment of nucleus-nucleus collisions is replaced by a simpler superposition model f , where the nucleus-nucleus collision is replaced by the corresponding nucleon-nucleus collisions. Here we study a model where the nucleus-nucleus collisions are treated in a more detailed way.

Soft and hard multiple interactions between nucleons of both nuclei dominate the hadron production in most of the kinematic region covered by the interaction and are well described in the framework of the Dual Parton Model (DPM) [3]. They were extensively studied with the Monte Carlo (MC) implementation of this model: DPMJET-II [4]. This event generator was already applied for sampling cosmic ray cascades $[4,5,6$. [. However, when dealing with particle or fragment production in the forward or backward fragmentation regions a detailed description of intranuclear cascade processes and of nuclear disintegration is important. A formation zone intranuclear cascade in the target nucleus was therefore considered since a few years [7, 8, 9, 4. A formation zone intranuclear cascade (FZIC) model in both the target as well as the projectile nucleus, the calculation of nuclear excitation energies, models for nuclear evaporation, high energy fission and break-up of light nuclei were discussed for hadron-nucleus and nucleus-nucleus collisions in [10, 11]. It was shown that these MC implementations for hadronnucleus and nucleus-nucleus interactions describe successfully the basic features of target and projectile associated particle production.

The model was compared in [10, 11] mostly to data for asymmetric collisions in which projectile and target masses are different. It seems that the performance of the model in these collisions is rather good. Asymmetric collisions are the most common ones also in cosmic ray cascades.

Here we will study this model with then aim to find differences with respect to the superposition models mentioned above.

In Section 2 we summarize briefly the main steps of sampling hadron-nucleus and nucleus-nucleus interactions within the event generator DPMJET-II. Furthermore, we summarize the basic ideas of the FZIC model. In Section 3 we calculate Feynman- $x_{F}$ distributions of pions produced in hadron-nucleus and nucleus-nucleus collisions. In Section 4 we study spectrum-weighted moments and energy fractions carried away by some kinds of secondaries and we compare them with the expectations from superposition models. It is pointed out that the

\footnotetext{
${ }^{1}$ it must be noticed that SIBYLL takes however into account the correct differences in the interaction heights as coming from the nucleus-nucleus cross sections.
} 
DPM differs from the superposition model. Finally, in Section 5 we summarize our results.

\section{The two-component DPM for hadron-nucleus and nucleus-nucleus collisions}

\subsection{The DPMJET-II event generator}

The two-component DPM and its MC realizations for hadron-hadron, hadronnucleus and nucleus-nucleus collisions have been discussed in detail in [12, 13, [4]. Therefore, we briefly summarize the main steps leading to the multiparticle state, which is the starting point for the intranuclear cascade.

The MC model for hadron-nucleus and nucleus-nucleus interactions starts from an impulse approximation for the nucleons of the interacting nuclei. The spatial initial configuration, i.e. the positions of the nucleons in space-time in the rest system of the corresponding nucleus, is sampled from standard density distributions. For energies above $3-5 \mathrm{GeV} /$ nucleon the collision proceeds via $\nu$ elementary interactions between $\nu_{p}$ and $\nu_{t}$ nucleons from the projectile and target, respectively. The values $\nu, \nu_{p}$, and $\nu_{t}$ are sampled according to Glauber's multiple scattering formalism using the $\mathrm{MC}$ algorithm of [14]. The particle production is well described by the two-component DPM which is applied as in hadron-hadron interactions [12, 13]. As a result, a system of chains connecting partons of the nucleons involved in the scattering process is formed. The chains are hadronized applying the JETSET model [15, [16]. The hadrons may then cause intranuclear cascade processes, which are treated by the FZIC model [0], an extension of the intranuclear cascade model of ref. [17, [18]. At energies below 3-5 GeV/nucleon the FZIC model is able by itself to describe reasonably the inelastic nuclear collisions.

\subsection{The formation zone cascade in target and projectile nuclei}

In the following we summarize the main ideas of the FZIC model for hadronnucleus and nucleus-nucleus interactions [7, 8, 9, 4, 10, 11]. The physical picture explaining the absence of the intranuclear cascade at high energies is the concept of the formation zone [19]. It has been introduced in analogy to the LandauPomeranchuk [20] effect, which explains the observation that electrons passing through high density materials become more penetrating at high energies. For the formation zone of an electron with 4-momentum $p$ and energy $E$ upon radiation of a photon with 4-momentum $k$ one obtains

$$
\tau=\frac{E}{k \cdot p}=\frac{E}{m} \frac{1}{\omega_{e}},
$$


where $\omega_{e}$ is the frequency of the photon in the rest frame of the electron and $E / m$ is the time dilatation factor from the electron rest frame to the laboratory. Within the quark model, the states being formed in the primary nucleon-nucleon interaction can be understood as consisting of valence quarks only (i.e without the full system of sea quarks, antiquarks and gluons) and have therefore a reduced probability for hadronic interactions inside the nucleus [7]. The formation zone concept can be translated to hadron production as follows [8]. We consider the formation zone cascade in the rest system of the target nucleus (laboratory system) or in the rest system of the projectile nucleus. Denoting the 4-momenta of the projectile hadron $p_{p}$ and of the secondary hadron $p_{s}$ with

$$
p_{p}=\left(E_{p}, 0,0, \sqrt{E_{p}^{2}-m_{p}^{2}}\right), \quad p_{s}=\left(E_{s}, \vec{p}_{s \perp}, \sqrt{E_{s}^{2}-m_{s}^{2}-\vec{p}_{s \perp}^{2}}\right)
$$

and replacing in Eq. (1) the electron momentum by $p_{p}$ and the photon momentum by $p_{s}$, the hadron formation time is, for $E_{p} \gg m_{p}$ :

$$
\tau_{\text {Lab }}=\frac{2 E_{s}}{\left(m_{p} x\right)^{2}+m_{s}^{2}+p_{s \perp}^{2}}, \quad x=\frac{E_{s}}{E_{p}} .
$$

The term $\left(m_{p} x\right)^{2}$ can be neglected for most of the produced secondaries, so one can approximate

$$
\tau_{\text {Lab }} \approx \gamma_{s} \tau_{s}, \quad \gamma_{s}=\frac{E_{s}}{m_{s}}
$$

We define an average formation time to create a complete hadronic state $\tau_{s}$ in the rest system of the secondary hadron $s$ [7, 8]:

$$
\tau_{s}=\tau_{0} \frac{m_{s}^{2}}{m_{s}^{2}+p_{s \perp}^{2}}
$$

where $\tau_{0}$ is a free parameter, which has to be determined by comparing particle production within the model to experimental data. Typical values are in the range from $1 \mathrm{fm} / c$ to $10 \mathrm{fm} / c$. Here we use $\tau_{0}=4.5 \mathrm{fm} / c$. This value differs somewhat from the values reported in ref. [10, 11]. The reason for this is an updated treatment of nuclear evaporation with respect to the version of the code used in [10, 11]. For each secondary we sample a formation time $\tau$ from an exponential distribution [21] with an average value as given in Eq. (5). As it was described in [9], in the MC model the full space-time history of the collision is known.

After having assigned a formation time to a secondary, its spatial coordinates in the rest system of both nuclei are known and we start with considering an intranuclear cascade step in one (randomly chosen) of the spectators.

Due to relativistic time dilatation, those secondaries which have sufficiently high energies in the rest frame of the considered nucleus are formed mostly outside of the spectator part of this nucleus, whereas those with lower energies are 
formed inside. The latter may penetrate the spectator and initiate intranuclear cascade processes. Elastic and inelastic interactions with spectator nucleons are treated using the MC-model HADRIN [22]. This code is based on measured cross sections and interaction channels up to a laboratory momentum of $5 \mathrm{GeV}$. We apply HADRIN to hadron-nucleon interactions up to $9 \mathrm{GeV}$ and neglect those at higher energies. Reinteractions beyond $5 \mathrm{GeV}$ occur much less frequently than reinteractions below $5 \mathrm{GeV}$ and a more detailed treatment would not change the results discussed in this paper. Furthermore, we take into account absorption of low-energy mesons and antiprotons by interactions with two-nucleon systems (for pion absorption we use the cross sections as given by Ritchie [23]) and Pauli's principle [9]. In case no interaction is possible in the considered spectator, we proceed with sampling a cascade step in another spectator.

For secondaries produced in intranuclear cascade processes we apply the same formalism, i.e. a formation time is sampled, the secondary is transported to the end of the formation zone and reinteractions are treated if they are possible. Due to these intranuclear cascade processes, nucleons are knocked out of the residual spectator nuclei if their energy is high enough to escape from the nuclear potential.

\section{Feynman- $-x_{F}$ distributions in hadron-nucleus and nucleus-nucleus collisions}

The so-called "cascade particles" are the particles which are produced or knockedout off the spectator nucleus by the FZIC. Target associated cascade particles are experimentally studied (mostly in emulsion experiments) and are often called "grey" prongs.

In the papers [10, 11] most of the available data on grey particle production in high energy hadron-nucleus and nucleus-nucleus collisions are compared to the model we use. Reasonable agreement is found as far as the average multiplicities of grey prongs, their multiplicity and angular distribution, and their correlations with the number of fast produced particles are concerned. There are two kinds of particles contributing to the grey prongs: protons and charged pions. The emulsion experiments are not able to differentiate between such protons and charged pions. There is however one heavy ion experiment (WA80 [24, 25]) which presents only protons in the energy region of the grey prongs in their multiplicity distributions. We can only draw some rather indirect support for the ability of our model to describe the pions from the intranuclear cascade. This support comes from the fact that the model agrees as well to the emulsion data on grey prongs as to the WA80 data on protons alone. Roughly $20 \%$ of the grey prongs (defined with Lorentz parameter $0.23<\beta<0.70$ ) in the model are charged pions 11.

The Feynman $-x_{F}$ distributions are the most suitable way to present the parti- 
cle production in the target and projectile fragmentation regions. Unfortunately, most experiments, which measure grey prongs are not able to measure the particle momenta and identity and are therefore not able to present Feynman- $x_{F}$ distributions. We define Feynman $-x_{F}$ in $\mathrm{h}-\mathrm{A}$ and $\mathrm{A}-\mathrm{A}$ collisions with the longitudinal momentum in the hadron-nucleon (or nucleon-nucleon) $\mathrm{cms} p_{z}^{c m s}$ :

$$
x_{F}=\frac{p_{z}^{c m s}}{\left|p_{z, \text { max }}^{c m s}\right|}
$$

where we use a $p_{z, \max }^{c m s}$ calculated disregarding Fermi momenta. The Feynman- $x_{F}$ distribution $f\left(x_{F}\right)$ is defined as follows

$$
f\left(x_{F}\right)=x_{F} \frac{d N}{d x_{F}} .
$$

For cosmic ray calculations we are mainly interested in the contributions of pions from the intranuclear cascade to the Feynman- $x_{F}$ distributions in the fragmentation region of the projectile nucleus, since particles from target fragmentation are very slow in the lab-frame. However, experimental data about the target fragmentation are very important for the tuning of the interaction model. The changes in Feynman- $x_{F}$ distributions from $\mathrm{p}-\mathrm{p}$ to $\mathrm{p}-$ nucleus collisions in the proton fragmentation region have been measured (and have been compared to DPMJET-II in [4]), but we are not aware of any such measurements in the fragmentation region of the nucleus. Therefore, we have to rely on the model, but we stress that it would be highly desirable to measure Feynman- $x_{F}$ distributions in the fragmentation region of target or projectile nuclei.

In Fig. 11 we present the Feynman- $x_{F}$ distribution of $\pi^{+}$mesons in p-air collisions at a lab-energy of $500 \mathrm{TeV}$. There are two plots, one for the full model with formation zone intranuclear cascade in the target nucleus, and one without this cascade. We also compare the distributions with the one in $\mathrm{p}-\mathrm{p}$ collisions at the same energy. In the target fragmentation region, at $x_{F}$ values between -0.3 and -1 , we find significant differences in the distributions due to the pions produced by the formation zone cascade in the target nucleus. The difference between the two plots is not due to a large number of particles. If we define "grey" the particles with Lorentz- $\beta$ of $0.23<\beta<0.70$ in the lab-frame, we find in the calculation 0.06 grey $\pi^{+}$per interaction, on average. However, not all charged pions from the formation zone cascade belong to the grey particles.

Next, we turn to nucleus-air collisions and calculate again Feynman- $x_{F}$ distributions. It is already enough to look at Fig.11 to understand, qualitatively, what happens in nucleus-nucleus collisions. Fig.1 gives the Feynman $x_{F}$ distribution in $\mathrm{N}$-p collisions if we simply exchange $x_{F}$ by $-x_{F}$. Therefore, we expect that the FZIC will lead to significant changes of the distributions in the fragmentation regions of nuclear projectiles in nucleus-proton and nucleus-nucleus collisions.

In Figs. 2 to 1 we present the Feynman $-x_{F}$ distribution of $\pi^{+}$mesons at a lab-energy of $500 \mathrm{TeV}$ per projectile nucleon in $\mathrm{He}$-air, $\mathrm{O}$-air, and $\mathrm{Fe}$-air 
collisions. The model, as shown in [4], exhibits a rather good Feynman-scaling behaviour, so that these distributions look rather similar also at other different energies. Fig. 2 is included mainly to demonstrate that for a light nucleus, like He, the formation zone cascade makes really no difference and can be neglected. There are two plots on each figure, one for the full model with formation zone intranuclear cascade in the target as well as in the projectile nucleus, and one without this cascade. We also compare the distributions with the corresponding p-air collisions at the same energy and with the formation zone cascade. In the target fragmentation region, at $x_{F}$ values between -0.3 and -1 we find in each plot nearly the same differences in the distributions due to the pions produced by the formation zone cascade in the target nucleus. Such differences are also visible in the projectile fragmentation region at positive $x_{F}$ values, but here those differences, which were not significant in the case of He-air collisions, are found to rise with the mass number of the projectile nucleus. Clearly, the formation zone cascade in the spectators of the Fe nucleus produces more pions than the one in the $\mathrm{O}$-spectators.

\section{Spectrum weighted moments, energy frac- tions and comparison with superposition mod- els}

Following for instance the basic discussion of Ref. [26], we introduce a variable $x_{l a b}$ similarly to Feynman- $x_{F}$, but this time in the lab-frame :

$$
x_{l a b}=\frac{E_{i}}{E_{0}},
$$

$E_{i}$ is the lab-energy of a secondary particle $i$ and $E_{0}$ is the lab-energy of the projectile in a $\mathrm{h}-\mathrm{A}$ collision (or the energy per projectile nucleon in a $\mathrm{A}-\mathrm{A}$ collision). We introduce $x_{l a b}$ distributions $F\left(x_{l a b}\right)$ :

$$
F_{i}\left(x_{l a b}\right)=x_{l a b} \frac{d N_{i}}{d x_{l a b}} .
$$

We note that the Feynman- $x_{F}$ distribution at positive $x_{F}$ in the projectile fragmentation region is a very good approximation to the $x_{l a b}$ distribution. Therefore, the Feynman $x_{F}$ distributions given in the last Section give also a rather good picture for $x_{l a b}$ distributions.

The cosmic ray spectrum weighted moments in A-B collisions are defined as moments of the $F\left(x_{l a b}\right)$

$$
Z_{i}^{A-B}=\int_{0}^{A_{A}}\left(x_{l a b}\right)^{\gamma-1} F_{i}^{A-B}\left(x_{l a b}\right) d x_{l a b} .
$$


Here $-\gamma \simeq-1.7$ is the power of the integral cosmic ray energy spectrum.

The spectrum weighted moments for nucleon-air collisions, as discussed in Ref. [26], determine the uncorrelated fluxes of energetic particles in the atmosphere. This result has been obtained for cosmic ray cascades initiated by a hadron primary. There, the spectrum weighted moments (which change only slightly with the primary energy) are the same for all generations of the cascade. We can not apply this result directly for our situation. A cosmic ray cascade initialized by primary nuclei becomes after the first two to three generations also a cascade with only hadrons participating. There are no theoretical arguments for the relevance of the spectrum weighted moments of nucleus-nucleus collisions for the cosmic ray cascade as a whole. These moments should be relevant only for the first generations. However, we might use the spectrum weighted moments and energy fractions just as any other moment of the Feynman- $x_{F}$ distribution with the aim to point out differences between models. In particular, we use these moments to stress that the superposition model for nucleus-nucleus collisions is in reality only a modest approximation to the correct treatment of nucleus-nucleus collisions.

We also introduce the energy fraction $K_{i}^{A-B}$. In $\mathrm{h}-\mathrm{A}$ collisions that is the fraction of primary energy carried by secondaries of type $i$; in $\mathrm{A}-\mathrm{B}$ collisions it is this energy fraction multiplied with the mass number $A$ of the projectile nucleus

$$
K_{i}^{A-B}=\int_{0}^{A_{A}} F_{i}^{A-B}\left(x_{l a b}\right) d x_{l a b},
$$

where $A_{A}$ is the mass number of the projectile nucleus A. After recalling the concept of superposition model, in the following we shall compare the calculation of spectrum weighted moments with our model in different conditions, pointing out the differences with respect to a simple superposition model.

\subsection{The superposition model}

Generally, we call superposition model the approximation in which in the cosmic ray cascade the collision of a nucleus $A$, with total energy $E$, against a target $B$, is treated as the superposition of $A_{A}$ independent nucleon-B collisions, each nucleon having an energy $\mathrm{E} / A_{A}$. This model is based on the hypothesis that, when the energy per nucleon of the projectile is much larger than the single nucleon binding energy, the $A_{A}$ nucleons will interact incoherently. In the context of cosmic ray simulations, for a primary of total energy $\mathrm{E}$ and mass number $A_{A}\left(A_{A}>1\right)$, the cascade generated is equivalent to the total effect of $A_{A}$ showers initiated by $A_{A}$ independent nucleons of energy $\mathrm{E} / A_{A}$. For example, the cascade for a primary iron nucleus of $560 \mathrm{TeV}$ would be simulated as the superposition of 26 proton + 30 neutron initiated showers, each of $10 \mathrm{TeV}$. Examples of application of the superposition model to cosmic ray calculations can be found in Ref. [27, 28]. 
Here, in the comparison with DPMJET-II we will apply a less general concept of superposition model which we call restricted superposition model, making reference to a single nucleus-nucleus interaction. In a minimum bias A-B collision, not all $A_{A}$ projectile nucleons interact. The number of interacting projectile nucleons is only $\nu_{p}^{A-B}$. For the collisions considered the average values of $\nu_{p}^{A-B}$ are always given in Tables 1 to 4 . Obviously, in order to make a comparison with DPMJET-II, we should use a restricted superposition model where only $\nu_{p}^{A-B}$ projectile nucleons interact. In this restricted superposition model we have for instance $Z_{\pi}^{s u p A-B}=\nu_{p}^{A-B} Z_{\pi}^{p-B}$ (for the same energy/nucleon of the projectile).

\subsection{The Glauber model in nucleus-nucleus collisions and the restricted superposition model}

The Glauber model for nucleus-nucleus collisions [29] is used by most models for nucleus-nucleus and nucleon-nucleus collisions. DPMJET uses the implementation of ref. [14]. This model has properties different from those of the restricted superposition model. In order to understand that, we recall the main formulae for inelastic nucleus-nucleus collisions. From these expressions we understand that the Glauber model cannot be reduced just to nuclear geometry.

We start with the scattering amplitude of two nuclei with mass numbers A and $\mathrm{B}$ in the impact parameter representation [30, 31, 32]

$$
F(\mathbf{b})_{A-B}=<\psi_{A}^{f} \psi_{B}^{f}\left|1-\prod_{j=1}^{A} \prod_{k=1}^{B}\left[1-\chi\left(\mathbf{b}-\mathbf{s}_{j}+\tau_{k}\right)\right]\right| \psi_{B}^{i} \psi_{A}^{i}>
$$

where $\mathbf{b}$ is the impact parameter vector and $\mathbf{s}_{j}$ and $\tau_{k}$ are the coordinates of the nucleons with respect to the centers of the nuclei $\mathrm{A}$ and $\mathrm{B}$, respectively, in the impact parameter plane. The $\psi_{A, B}^{i}$ and $\psi_{A, B}^{f}$ are the initial and final state wave functions of nuclei $\mathrm{A}$ and $\mathrm{B}$, while $\chi(\mathbf{b})$ is the dimensionless elastic nucleon-nucleon scattering amplitude in the impact parameter representation. The (Gaussian) parametrization of $\chi(\mathbf{b})$ used in DPMJET-II was described in detail in 4 .

Furthermore, we use a simple assumption for the squares of the state wave functions

$$
\left|\psi_{A}\right|^{2}=\prod_{j=1}^{A} \rho_{A}\left(\mathbf{s}_{j}, z_{j}\right), \quad\left|\psi_{B}\right|^{2}=\prod_{k=1}^{B} \rho_{B}\left(\tau_{k}, \xi_{k}\right)
$$

where $\rho_{A}$ and $\rho_{B}$ are the one particle densities of the nuclei A and B.

From this we get the total A-B cross section

$$
\sigma_{A-B}^{t o t}=2 R e \int d^{2} \mathbf{b} F(\mathbf{b})_{A-B} .
$$

The inelastic $\mathrm{A}-\mathrm{B}$ cross section is determined by the profile function $\Gamma(\mathbf{b})$

$$
\sigma_{A-B}^{i n e l}=\int d^{2} \mathbf{b} \Gamma(\mathbf{b})
$$


$\Gamma(\mathbf{b})$ is defined as follows

$$
\Gamma(\mathbf{b})=\int\left\{1-\prod_{i=1}^{A} \prod_{j=1}^{B}\left(1-p_{i j}\right)\right\}\left\{\prod_{i=1}^{A} \rho_{A}\left(\mathbf{r}_{i}\right) d^{3} \mathbf{r}_{i}\right\}\left\{\prod_{j=1}^{B} \rho_{B}\left(\mathbf{t}_{j}\right) d^{3} \mathbf{t}_{j}\right\}
$$

where

$$
p_{i j}=\chi\left(\mathbf{b}-\mathbf{s}_{i}+\tau_{j}\right)+\chi^{*}\left(\mathbf{b}-\mathbf{s}_{i}+\tau_{j}\right)-\chi\left(\mathbf{b}-\mathbf{s}_{i}+\tau_{j}\right) \chi^{*}\left(\mathbf{b}-\mathbf{s}_{i}+\tau_{j}\right) .
$$

This profile function, which contains more than the nuclear geometry, is also used to sample in the Monte Carlo calculation the impact parameters of the inelastic A-B collisions.

Sampling the impact parameters of nucleon-nucleus and nucleus-nucleus collisions simply from geometry, will certainly lead to a restricted superposition model. Unfortunately, it is somewhat cumbersome to see, without the actual numerical calculations, that the profile functions according to the Glauber model for nucleon-nucleus collisions differ sufficiently from the profile functions for nucleusnucleus collisions to prevent the restricted superposition model to give identical results as the full model. As an example related to the case discussed in the next subsection, we show in Fig. 5 the profile functions for p-Nitrogen $\mathrm{He}-\mathrm{Nitrogen}$ and $\mathrm{Fe}-$ Nitrogen as function of $b=|\mathbf{b}|$. For p-Nitrogen collisions, the profile function never becomes completely absorptive $(\Gamma(b)=1)$, even in the center $(b=0)$, while in Fe-Nitrogen collisions $\Gamma(b)=1$ is reached for $b<5 \mathrm{fm}$.

\subsection{Calculation results, DPMJET-II without FZIC, eva- poration and determination of the residual nuclei}

In Table 1 and 2 we present spectrum weighted moments and energy fractions as calculated in DPMJET-II in the model without FZIC, evaporation and consequent determination of the residual nuclei. We consider p-air, He-air, O-air and $\mathrm{Fe}$-air collisions at laboratory energies per nucleon of $50 \mathrm{GeV}, 500 \mathrm{TeV}$, and $5 \cdot 10^{6} \mathrm{TeV}$, the latter energy $\left(5 \cdot 10^{18} \mathrm{eV}\right)$ is the absolute highest energy at which DPMJET-II is valid, at present. We also give the average number of projectile nucleons $\nu_{p}$ participating in minimum bias collisions.

In Tables 11 and 2 we present a further comparison, at the two upper energies, of the spectrum weighted moments of pions and kaons and the energy fractions of pions, kaons and baryons minus antibaryons according to the model, with the moments and energy fractions obtained from the restricted superposition model. The baryons are here only the secondary baryons from the collision. Spectator baryons from the projectile or target nucleus do not contribute to the energy

fraction $K_{b-\bar{b}}^{A-B}$. Since the energy fraction of the newly produced baryons (produced in $b \bar{b}$ pairs) is equal to the energy fraction of the produced antibaryons, 
the energy fraction $K_{b-\bar{b}}^{A-B}$ gives the energy fraction retained by the baryons participating in the collision. In the restricted superposition model the spectrum weighted moments and energy fractions are given by the simple expressions

$$
\begin{aligned}
& Z_{i}^{\text {sup } A-B}=\nu_{p}^{A-B} Z_{i}^{N-B}, \\
& K_{i}^{\text {sup } A-B}=\nu_{p}^{A-B} K_{i}^{N-B},
\end{aligned}
$$

where $\mathrm{N}-\mathrm{B}$ refers to a nucleon nucleus collision.

We know, without any calculation, that the Dual Parton Model for nucleusnucleus collisions differs from the restricted superposition model. There are at least three reasons for these differences:

1. Properties of the Glauber cascade: The deviations of the Glauber model from the restricted superposition model were already discussed in the previous subsection. In Table 3 we give for each collision considered the average numbers of collisions according to the Glauber cascade. $\nu, \nu_{p}$ and $\nu_{t}$ were already explained above. In nucleon-nucleus collisions we have always $\nu^{N-B}$ $=\nu_{t}^{N-B}$ and $\nu_{p}^{N-B}=1$. In $\mathrm{A}-\mathrm{B}$ collisions we have $\nu^{A-B}, \nu_{t}^{A-B}$ and $\nu_{p}^{A-B}$ all different from one. In the $\mathrm{A}-\mathrm{B}$ collisions, a projectile nucleon interacts, in average, $N_{p}^{A-B}=\nu^{A-B} / \nu_{p}^{A-B}$ times. As seen from Table 3, $N_{p}^{A-B}$ is always different from $\nu_{t}^{N-B}$ at the same energy, $N_{p}^{A-B}$ being smaller than the corresponding $\nu_{t}^{N-B}$. The average nucleon-nucleus collision in an A-B collision has different properties compared to an average nucleon-nucleus collision. This is valid for any model constructed on the basis of the Glauber cascade.

2. We turn to the DPM with Glauber cascade, and let us for simplicity discuss the chain structure disregarding the multiple soft chains and multiple minijets in each nucleon-nucleon interaction, which are in addition present in each elementary Glauber collision. We construct in nucleon-nucleus collisions one pair of valence-valence chains and $\nu_{t}^{N-B}-1$ pairs of sea-valence chains. In the restricted superposition model for the $\mathrm{A}-\mathrm{B}$ collision we obtain $\nu_{p}^{A-B}$ pairs of valence-valence chains and $\nu_{p}^{A-B}\left(\nu_{t}^{N-B}-1\right)$ pairs of sea-valence chains.

In A-B collisions we have $\nu^{A-B}, \nu_{t}^{A-B}$ and $\nu_{p}^{A-B}$ all different from one and in Fe-Air collisions we have usually $\nu^{A-B}>\nu_{p}^{A-B}>\nu_{t}^{A-B}$. In this situation we might form $\nu_{t}^{A-B}$ valence-valence chain pairs, $\nu_{p}^{A-B}-\nu_{t}^{A-B}$ valencesea chain pairs and $\nu^{A-B}-\nu_{p}^{A-B}$ sea-sea chain pairs (this is the simplest possibility, in actual Monte Carlo models like DPMJET-II the chain ends available are connected in a random way). We know already that only the number of valence-valence chain pairs in the restricted superposition model and in the correct model correspond to each other, the number of sea-valence chain pairs will be different. Furthermore, there are never any sea-sea chains in the restricted superposition model. 
3. The single diffractive cross sections in hadron-nucleus collisions are well studied [33, 34] and included in the model. The cross sections for single diffractive nucleus-nucleus collisions are straightforward to calculate within the DPM, and they will probably have a size similar to that obtained in hadron-nucleus collisions. The $\mathrm{O}$-air or Fe-air total cross sections are three to five times larger than the p-air total cross section. Therefore, the fraction of single diffractive nucleus-nucleus collisions will come out to be a few times smaller than in hadron-nucleus collisions. For these reasons, and because of the lack of experimental data on single diffractive nucleus-nucleus cross sections, this component is not included at present in the DPMJET-II event generator (however, the inclusion of this part would probably not change the situation). Now, in the case of diffractive p-air collisions, where the projectile proton leaves the collision only slightly deflected and in which hadrons are produced only in the nucleus fragmentation region, nearly no contribution to the moments of pions and kaons is achieved. Therefore, also this effect is expected to lead to larger moments of charged pions and kaons in the correct DPM with respect to the restricted superposition model.

Looking at Tables 1 and 2 we find our expectations fulfilled. The spectrum weighted moments of pions and kaons according to DPMJET-II are 10 to $20 \%$ larger than the ones according to the restricted superposition model. Also the energy fractions carried by pions and kaons are for He-air and $\mathrm{O}-$ air about 5 to $10 \%$ larger than in the restricted superposition model. In Fe-air these energy fractions behave in a more complicated way. In the Fe-air reaction, it would be better to define the restricted superposition model in the Fe rest frame with the air nuclei as projectiles. At the same time, as expected, the energy fractions carried by the participating nucleons are for Fe-air and O-air in DPMJET-II always smaller than in the restricted superposition model, i.e. more energy is used for particle creation. Here the numbers obtained in He-air are more difficult to understand. We have to remember that all numbers in the Tables are the result of Monte Carlo calculations with typically $5 \%$ statistical error.

In summary, we may conclude that for the model without FZIC we find an agreement with the restricted superposition model within approximately 10 to $20 \%$.

\subsection{Calculation results, the full DPMJET-II model}

In Table 3 and 4 we present spectrum weighted moments and energy fractions as calculated in the full DPMJET-II model. We consider again p-air, He-air, O-air and $\mathrm{Fe}$-air collisions at laboratory energies per nucleon of $50 \mathrm{GeV}, 500 \mathrm{TeV}$, and $5 \cdot 10^{6} \mathrm{TeV}$. We also give the number of Glauber collisions $\nu$ between $\nu_{p}$ projectile and $\nu_{t}$ target nucleons in minimum bias collisions in Table 3 .

In Tables 3 and 4 we have a further comparison, at two energies, of the spec- 
trum weighted moments of pions and kaons and of the energy fractions of charged pions, charged kaons, baryons minus antibaryons and of nuclear fragments and residual nuclei, with the moments obtained from the restricted superposition model. Here we have to use slightly different expressions for the energy fractions in the restricted superposition model. The reason is that all spectators in the full DPMJET-II appear among the final state particles, either as evaporation protons or neutrons or as nuclear fragments and residual nuclei. We are not able to define nuclear fragments and residual nuclei in the restricted superposition model. In this respect the features of DPMJET-II are again different from those of the restricted superposition model. We use the expressions for moments and energy fractions for charged pions and kaons in the same form as given in the last subsection. For the energy fraction of $b-\bar{b}$ we use:

$$
K_{b-\bar{b}}^{s u p} A-B=\nu_{p}^{A-B} K_{b-\bar{b}}^{N-B}+\left(A_{A}-\nu_{p}^{A-B}\right) \cdot 1 .
$$

Since we are not able to define an energy fraction $K_{n . f}$. for nuclear fragments, we should compare the $K_{b-\bar{b}}^{\text {sup } A-B}$ in Table 4 with the sum of $K_{b-\bar{b}}^{A-B}$ and $K_{n . f .}^{A-B}$.

The differences between the spectrum weighted moments of the pions in the restricted model and in the full model in Tables 1 and 3 are easy to understand looking at the previously presented Feynman $-x_{F}$ distributions in the projectile fragmentation regions. Comparing the results of DPMJET-II with full FZIC with the restricted superposition model in Tables 3 and 4 we find:

1. For He-air collisions, and presumably for all light nuclei, the full model agrees practically to the model without formation zone cascade. Therefore, we also find only a $20 \%$ disagreement with the restricted superposition model.

2. We find that, for $\mathrm{O}$-air and $\mathrm{Fe}$-air, differences in the spectrum weighted moments of pions and kaons are up to $50-70 \%$ with respect to the restricted superposition model. We understand the reasons for this large disagreement looking at the changes in the Feynman $x_{F}$ distributions due to the FZIC as discussed in the last Section. The same trend is seen in the energy fractions of pions and kaons, but there the differences are only around $10 \%$.

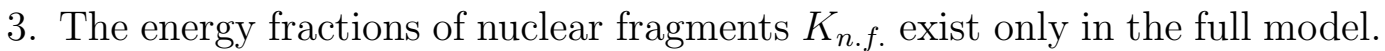
We find however the sum $K_{b-\bar{b}}+K_{n . f}$. of the full model remarkably close to the energy fractions $K_{b-\bar{b}}^{\text {sup }}$ of the restricted superposition model.

\section{Conclusions and summary}

We have compared Feynman- $x_{F}$ distributions, spectrum weighted moments and energy fractions of pions, kaons and baryons minus antibaryons in two versions of the two component Dual Parton Model. One version is the full model with 
FZIC of the produced hadrons with the spectators of the target and projectile nuclei, the second version is the DPM (closer to a restricted superposition model) without this formation zone cascade, nuclear evaporation and the formation of a residual nucleus.

We have discussed the reasons for deviations expected between the DPM and restricted superposition models. Nevertheless, we find a reasonable, however not perfect, agreement of the moments from the restricted model for nucleus-nucleus collisions with a restricted superposition model.

In the full model instead, the hadrons produced in the projectile fragmentation region by the formation zone cascade lead to significant deviations in the pion spectrum weighted moments from the restricted superposition model. For Fe-air collisions this difference becomes as large as $70 \%$. This effect depends on the changes in the Feynman- $x_{F}$ distributions due to the cascade in the projectile nucleus. We have stressed above that there is no direct experimental evidence for this. Therefore we cannot be completely sure about the quantitative size of the predicted effect, but we are convinced that this effect exists.

These differences are certainly significant and could also lead to differences when sampling the cosmic ray cascade with the full DPMJET-II model as compared to the sampling using the superposition model. Larger values for $Z_{\pi}$ and $K_{\pi}$ (also for $\pi^{0}$, where the differences are similar, and kaons) mean that, on the average, the primary cosmic ray loses a larger fraction of its energy in the first interaction. This energy is spent for pion production. On the other hand, we expect that less energy is carried away by the leading particles, so that the development of the shower might be different from that resulting from the superposition model. Depending on the relevance of the first interaction, this should probably lead to showers with a larger muon content and with a smaller depth of the maximum development (thus with a smaller electromagnetic size at mountain or sea level). We might also expect that in the simulation of the full cosmic ray cascade initiated by primary nuclei, the full model could well show further deviations from the superposition model which are not simply contained in the differences of spectrum weighted moments and energy fractions of pions and kaons. This is another reason to stress the importance of studying the full model in shower simulations.

In summary, DPMJET-II including FZIC offers now the opportunity to perform a true quantitative test of the validity (or failure) of the superposition approximation. A detailed study of the effects of the FZIC model on cosmic ray showers as a function of the primary energy and mass will be the object of a next paper.

\section{Acknowledgements}

We thank T.K.Gaisser and P.Lipari for useful discussions. One of the authors (J.R.) thanks Professor C.Pajares for the hospitality at Santiago de Compostela and he was supported by the Direccion General de Politicia Cientifica of Spain. 


\section{References}

[1] C. Forti, H. Bilokon, B. d'Ettorre Piazzoli, T.K. Gaisser, L. Satta and T. Stanev: Phys. Rev. D42 (1990) 3668

[2] R.S. Fletcher, T.K. Gaisser, P. Lipari and T. Stanev,: Phys. Rev. D50 (1994) 5710

[3] A. Capella, U. Sukhatme, C. I. Tan and J. Tran Thanh Van: Phys. Rep. 236 (1994) 227

[4] J. Ranft: Phys. Rev. D51 (1995) 64

[5] G. Battistoni, C. Forti and J. Ranft: Astroparticle Phys. 3 (1995) 157

[6] G. Battistoni, C. Bloise, C. Forti, M. Greco, J. Ranft and A. Tanzini: Astroparticle Physics 4 (1996) 351

[7] J. Ranft: Phys. Rev. D37 (1988) 1842

[8] J. Ranft: Z. Phys. C43 (1989) 439

[9] H.-J. Möhring and J. Ranft: Z. Phys. C52 (1991) 643

[10] A. Ferrari, J. Ranft, S. Roesler and P. R. Sala: Z. Phys. C70 (1996) 413

[11] A. Ferrari, J. Ranft, S. Roesler and P. R. Sala: Z. Phys. C71 (1996) 75

[12] P. Aurenche, F. W. Bopp, A. Capella, J. Kwiecinski, M. Maire, J. Ranft and J. Tran Thanh Van: Phys. Rev. D45 (1992) 92

[13] F. W. Bopp, R. Engel, D. Pertermann and J. Ranft: Phys. Rev. D49 (1994) 3236

[14] S. Y. Shmakov, V. V. Uzhinskii and A. M. Zadoroshny: Comp. Phys. Commun. 54 (1989) 125

[15] T. Sjöstrand: Comp. Phys. Commun. 39 (1986) 347

[16] T. Sjöstrand and M. Bengtsson: Comp. Phys. Commun. 43 (1987) 367

[17] H. W. Bertini: Phys. Rev. 137 (1963) 1801

[18] H. W. Bertini: Phys. Rev. 188 (1969) 1711

[19] L. Stodolski: Proceedings on the Colloquium on Multiparticle Reactions, Oxford (1975) 577

[20] L. Landau and I. Pomeranchuk: Dokl. Akad. Nauk SSR 92 (1953) 535,734 
[21] A. Bialas: Z. Phys. C26 (1984) 301

[22] K. Hänssgen and J. Ranft: Comp. Phys. Commun. 39 (1986) 37

[23] B. G. Ritchie: Phys. Rev. C 28 (1983) 926

[24] WA80 Collab.: R. Albrecht et al.: Z. Phys. C57 (1993) 37

[25] WA80 Collab.: R. Albrecht et al.: Phys. Lett. B309 (1993) 269

[26] T. K. Gaisser: Cosmic Rays and Particle Physics, Cambridge University Press, 1990

[27] J.W. Elbert, T.K. Gaisser and T. Stanev: Phys. Rev. D27 (1983) 1448

[28] T. K. Gaisser and T. Stanev: Nuclear Instr. \& Meth. A235 (1985) 183

[29] R. Glauber: in Lectures in Theoretical Physics, eds. W.E.Brittin et al., vol 1 p. 315 Interscience New York 1959

[30] V. Franco: Phys. Rev. 175 (1968) 1376

[31] W. Czyz and L. Maximon: Ann. of Phys. (NY) 52 (1969) 59

[32] O. Kofoed-Hansen: Nuovo Cim. 60A (1969) 621

[33] S. Roesler, R. Engel and J. Ranft: Z. Phys. C59 (1993) 481

[34] J. Ranft and S. Roesler: Z. Phys. C62 (1994) 329 
Table 1: Spectrum weighted moments of pions and kaons according to DPMJET-II in the model without FZIC. In the last columns we compare with the restricted superposition model. $E$ is the lab-energy per projectile nucleon.

\begin{tabular}{|c|c|c|c|c|c|c|}
\hline Collision & $\mathrm{E}(\mathrm{TeV})$ & $Z_{\pi}$ & $Z_{K}$ & $\nu_{p}$ & $\nu_{p} Z_{\pi}^{p-a i r}$ & $\nu_{p} Z_{K}^{p-a i r}$ \\
\hline p-air & 500 & 0.0640 & 0.0092 & 1 & & \\
& $5 \cdot 10^{6}$ & 0.0595 & 0.0087 & 1 & & \\
\hline He-air & 500 & 0.178 & 0.025 & 2.32 & 0.148 & 0.021 \\
& $5 \cdot 10^{6}$ & 0.180 & 0.026 & 2.60 & 0.155 & 0.023 \\
\hline O-air & 500 & 0.425 & 0.059 & 5.56 & 0.356 & 0.051 \\
& $5 \cdot 10^{6}$ & 0.462 & 0.065 & 6.49 & 0.386 & 0.057 \\
\hline Fe-air & 500 & 0.912 & 0.124 & 11.61 & 0.743 & 0.107 \\
& $5 \cdot 10^{6}$ & 1.020 & 0.128 & 14.46 & 0.860 & 0.126 \\
\hline
\end{tabular}


Table 2: Energy fractions of pions, kaons and baryons minus antibaryons according to DPMJET-II in the model without FZIC. In the last columns we compare with the restricted superposition model. $E$ is the lab-energy per projectile nucleon.

\begin{tabular}{|c|c|c|c|c|c|c|c|c|}
\hline Collision & $\mathrm{E}(\mathrm{TeV})$ & $K_{\pi}$ & $K_{K}$ & $K_{b-\bar{b}}$ & $\nu_{p}$ & $\nu_{p} K_{\pi}^{p-a i r}$ & $\nu_{p} K_{K}^{p-\text { air }}$ & $\nu_{p} K_{b-\bar{b}}^{p-\text { Air }}$ \\
\hline p-air & 500 & 0.336 & 0.048 & 0.268 & 1 & & & \\
& $5 \cdot 10^{6}$ & 0.361 & 0.052 & 0.193 & 1 & & & \\
\hline He-air & 500 & 0.89 & 0.13 & 0.82 & 2.32 & 0.78 & 0.11 & 0.62 \\
& $5 \cdot 10^{6}$ & 1.01 & 0.14 & 0.76 & 2.60 & 0.94 & 0.14 & 0.50 \\
\hline O-air & 500 & 2.02 & 0.28 & 1.22 & 5.56 & 1.87 & 0.27 & 1.49 \\
& $5 \cdot 10^{6}$ & 2.43 & 0.32 & 1.24 & 6.49 & 2.34 & 0.34 & 1.25 \\
\hline Fe-air & 500 & 4.05 & 0.54 & 2.37 & 11.61 & 3.90 & 0.56 & 3.11 \\
& $5 \cdot 10^{6}$ & 4.70 & 0.60 & 2.35 & 14.46 & 5.22 & 0.75 & 2.79 \\
\hline
\end{tabular}


Table 3: Spectrum weighted moments of pions and kaons according to DPMJET-II in the model with full FZIC. We give also the values of $\nu, \nu_{p}$ and $\nu_{t}$ for the minimum bias Glauber cascade introduced in Section 2.1. $E$ is the labenergy per projectile nucleon. In the last columns we compare with the restricted superposition model.

\begin{tabular}{|c|c|c|c|c|c|c|c|c|}
\hline Collision & $\mathrm{E}(\mathrm{TeV})$ & $\nu$ & $\nu_{p}$ & $\nu_{t}$ & $Z_{\pi}$ & $Z_{K}$ & $\nu_{p} Z_{\pi}^{p-a i r}$ & $\nu_{p} Z_{K}^{p-a i r}$ \\
\hline p-air & 500 & 2.05 & 1. & 2.05 & 0.0640 & 0.0091 & & \\
& $5 \cdot 10^{6}$ & 2.73 & 1. & 2.73 & 0.0586 & 0.0085 & & \\
\hline He-air & 500 & 4.44 & 2.32 & 3.41 & 0.182 & 0.026 & 0.148 & 0.021 \\
& $5 \cdot 10^{6}$ & 6.58 & 2.60 & 4.37 & 0.175 & 0.025 & 0.152 & 0.022 \\
\hline O-air & 500 & 10.34 & 5.55 & 5.32 & 0.499 & 0.065 & 0.355 & 0.051 \\
& $5 \cdot 10^{6}$ & 14.35 & 6.22 & 5.81 & 0.522 & 0.065 & 0.364 & 0.053 \\
\hline Fe-air & 500 & 21.51 & 11.89 & 6.87 & 1.274 & 0.137 & 0.761 & 0.101 \\
& $5 \cdot 10^{6}$ & 32.48 & 13.86 & 7.25 & 1.287 & 0.137 & 0.812 & 0.118 \\
\hline
\end{tabular}


Table 4: Energy fractions of pions, kaons, baryons minus antibaryons and nuclear fragments according to DPMJET-II in the model with full FZIC. We give also the values of $\nu_{p}$ for the minimum bias Glauber cascade introduced in Section 2.1. $E$ is the lab-energy per projectile nucleon. In the last columns we compare with the restricted superposition model.

\begin{tabular}{|c|c|c|c|c|c|c|c|c|c|}
\hline Collision & $\mathrm{E}(\mathrm{TeV})$ & $\nu_{p}$ & $K_{\pi}$ & $K_{K}$ & $K_{b-\bar{b}}$ & $K_{n . f .}$ & $\nu_{p} K_{\pi}^{p-a i r}$ & $\nu_{p} K_{K}^{p-a i r}$ & $K_{b-\bar{b}}^{\text {sup }}$ \\
\hline p-air & 500 & 1. & 0.35 & 0.049 & 0.25 & & & & \\
& $5 \cdot 10^{6}$ & 1. & 0.37 & 0.053 & 0.18 & & & & \\
\hline He-air & 500 & 2.32 & 0.90 & 0.13 & 1.58 & 0.52 & 0.81 & 0.11 & 2.26 \\
& $5 \cdot 10^{6}$ & 2.60 & 0.99 & 0.14 & 1.37 & 0.49 & 0.95 & 0.14 & 1.87 \\
\hline O-air & 500 & 5.55 & 2.22 & 0.29 & 4.35 & 7.36 & 1.93 & 0.27 & 11.84 \\
& $5 \cdot 10^{6}$ & 6.21 & 2.56 & 0.33 & 4.11 & 7.17 & 2.27 & 0.33 & 10.91 \\
\hline Fe-air & 500 & 11.89 & 4.81 & 0.57 & 18.01 & 28.19 & 4.14 & 0.58 & 47.08 \\
& $5 \cdot 10^{6}$ & 13.86 & 5.43 & 0.63 & 16.03 & 28.92 & 5.06 & 0.73 & 44.63 \\
\hline
\end{tabular}




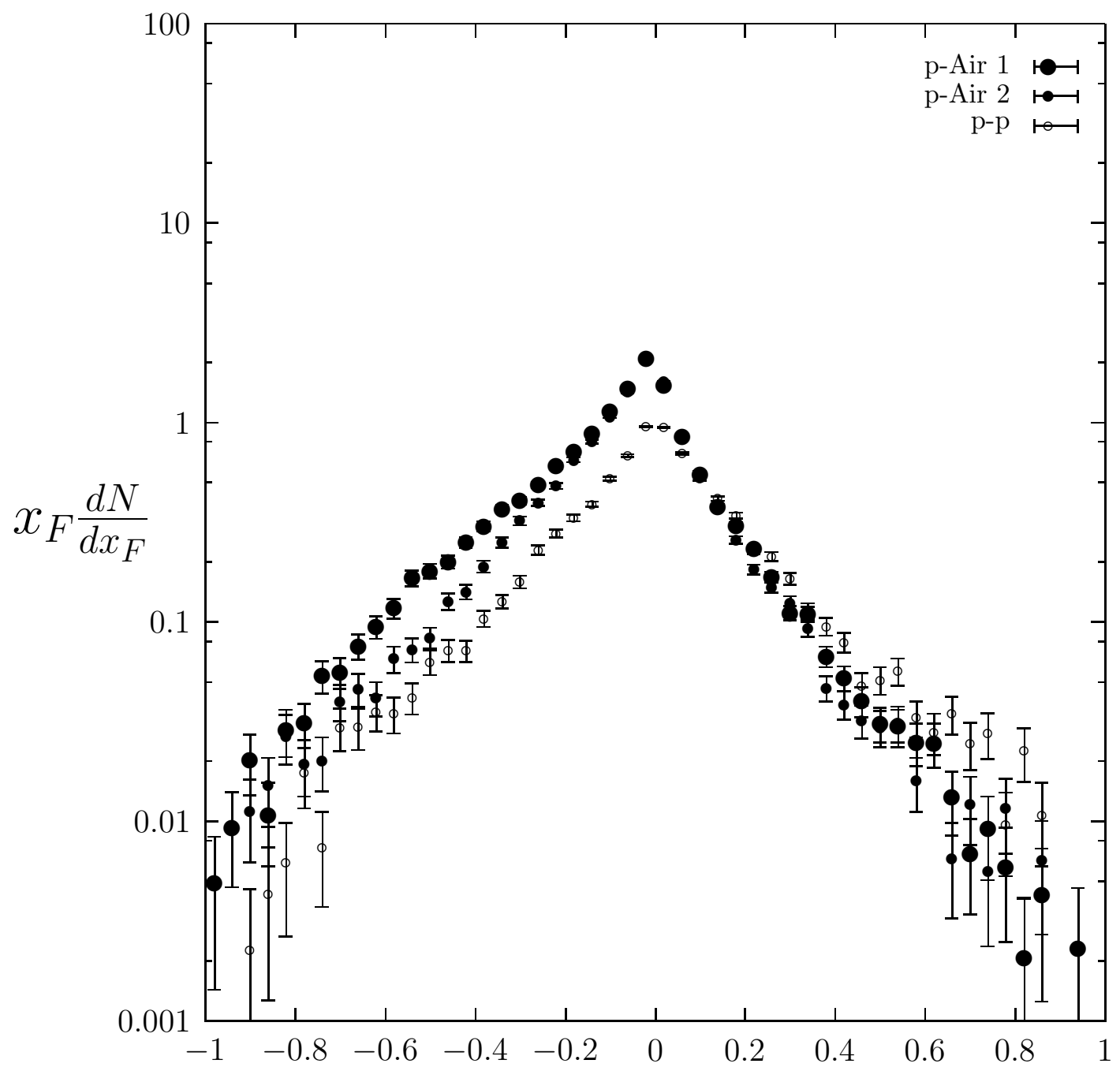

Feynman $x_{F}$

Figure 1: Feynman- $x_{F}$ distribution of $\pi^{+}$in p-air collisions at a lab-energy of $500 \mathrm{TeV}$. Plot 1 (large full circles) is for the full model with FZIC in the target nucleus; plot 2 (small full circles) is for the model without FZIC. The target fragmentation region is at negative Feynman $-x_{F}$, the projectile fragmentation is at positive $x_{F}$. For comparison we give also the same distribution in $\mathrm{p}-\mathrm{p}$ collisions (small empty circles). 


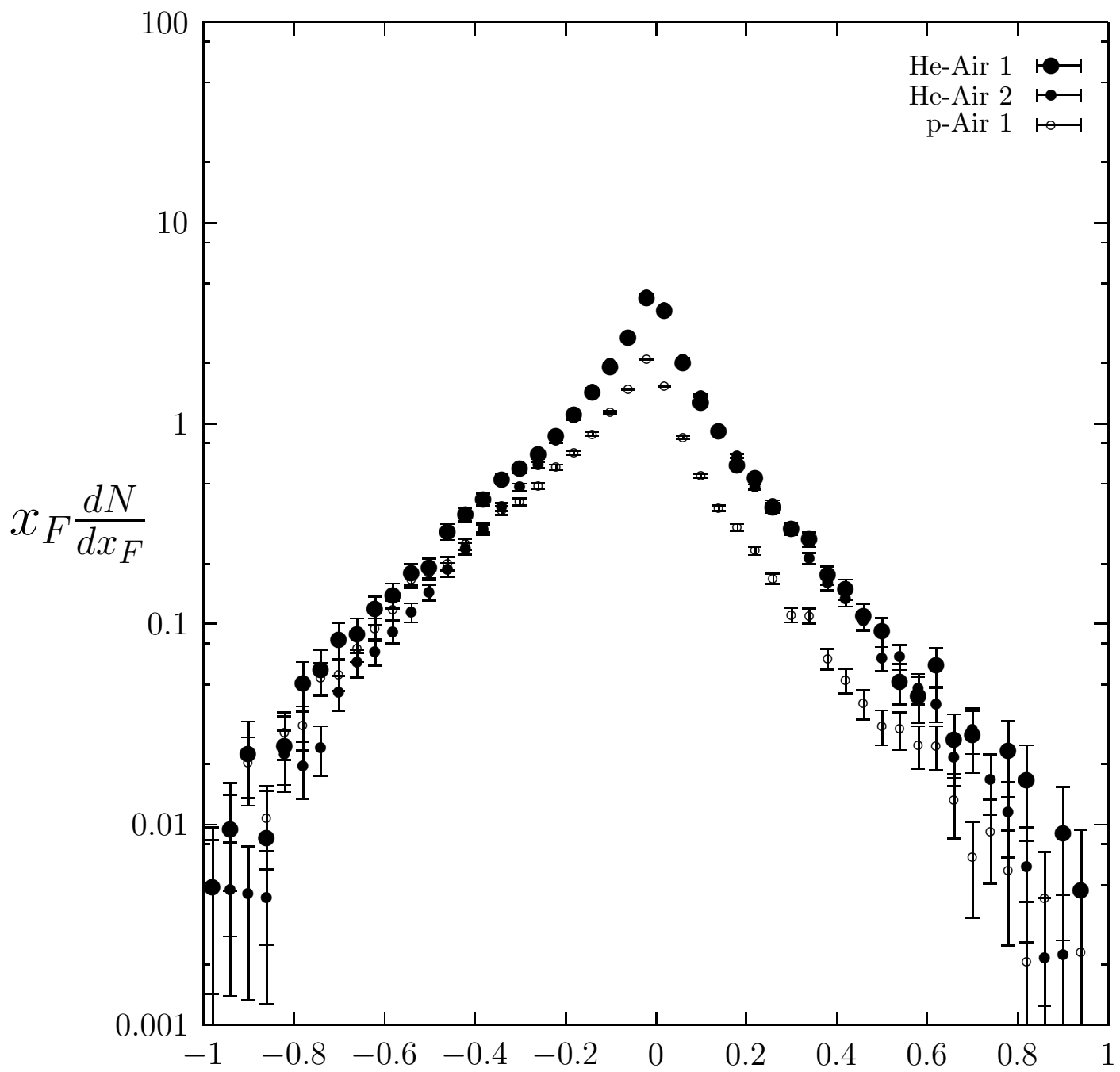

Feynman $x_{F}$

Figure 2: Feynman- $x_{F}$ distribution of $\pi^{+}$in He-air collisions at a lab-energy of $500 \mathrm{TeV}$ per projectile nucleon. Plot 1 (large full circles) is for the full model with FZIC in the target nucleus, and projectile nucleus; plot 2 (small full circles) is for the model without FZIC. The target fragmentation region is at negative Feynman- $x_{F}$, the projectile fragmentation is at positive $x_{F}$. For comparison we give also the same distribution in p-air collisions with FZIC (small empty circles). 


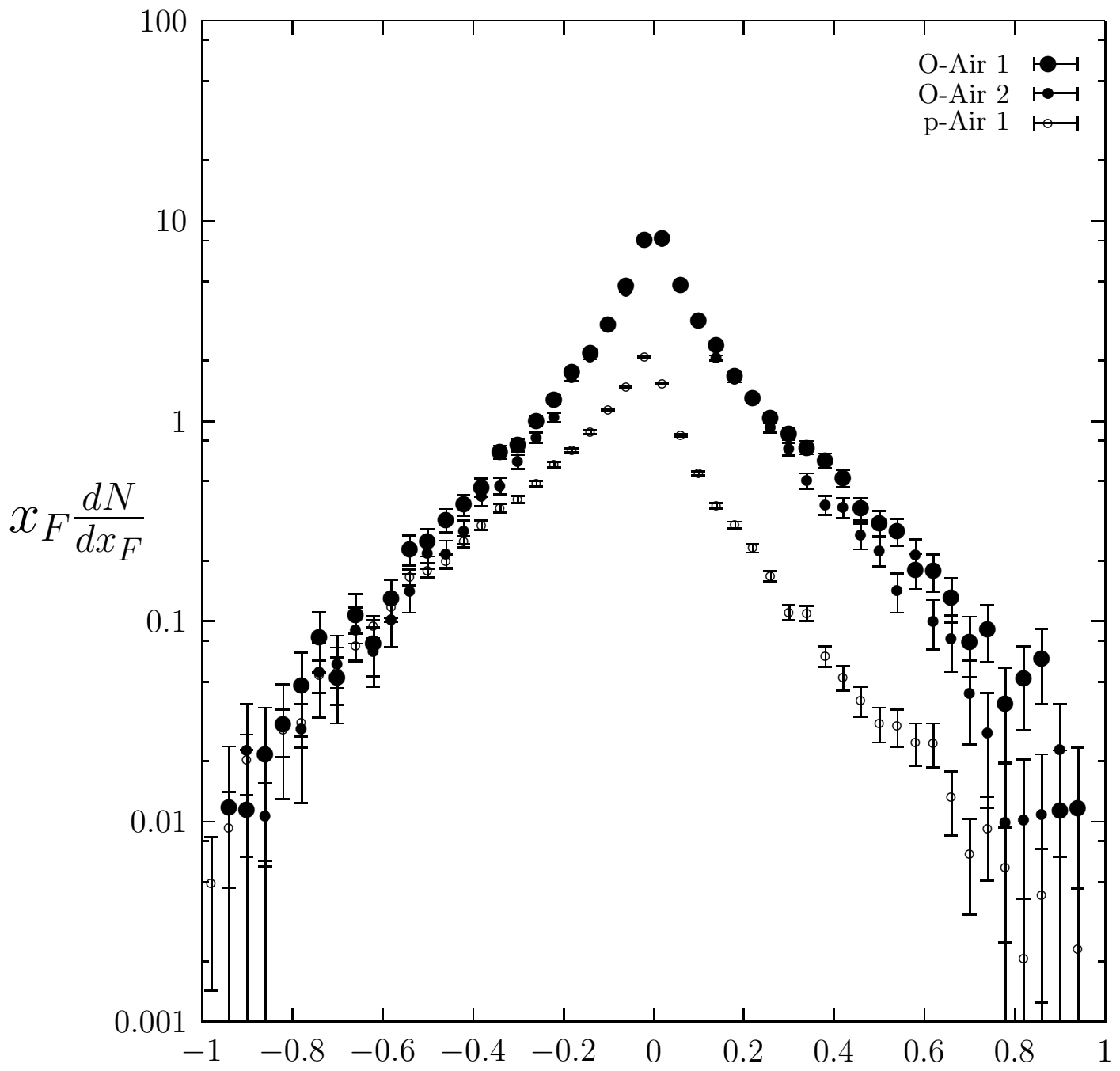

Feynman $x_{F}$

Figure 3: Feynman- $x_{F}$ distribution of $\pi^{+}$in $\mathrm{O}$-air collisions at a lab-energy of $500 \mathrm{TeV}$ per projectile nucleon. Plot 1 (large full circles) is for the full model with FZIC in the target nucleus, and projectile nucleus; plot 2 (small full circles) is for the model without FZIC. The target fragmentation region is at negative Feynman- $x_{F}$, the projectile fragmentation is at positive $x_{F}$. For comparison we give also the same distribution in p-air collisions with FZIC (small empty circles). 


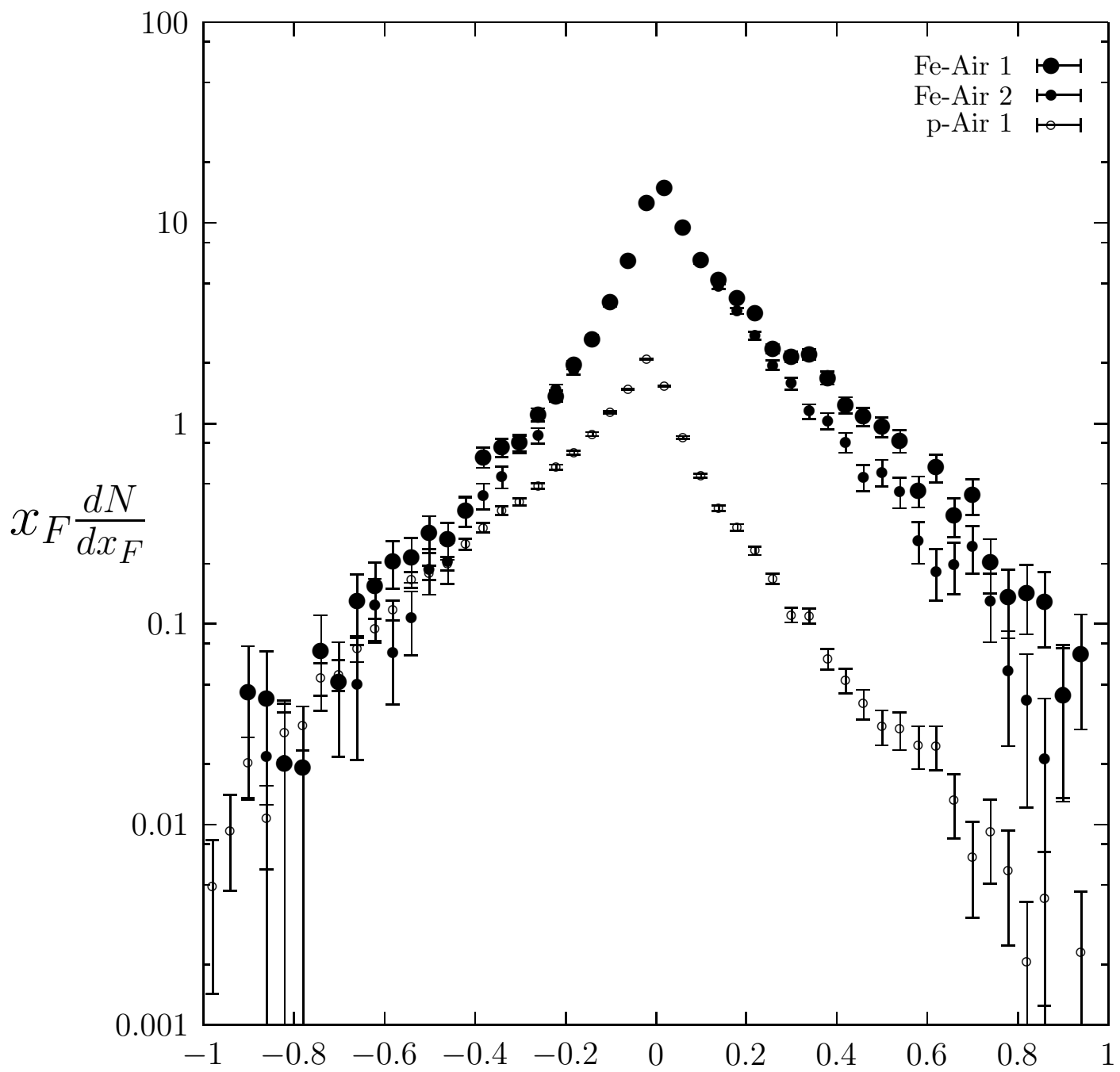

Feynman $x_{F}$

Figure 4: Feynman- $x_{F}$ distribution of $\pi^{+}$in Fe-air collisions at a lab-energy of $500 \mathrm{TeV}$ per projectile nucleon. Plot 1 (large full circles) is for the full model with FZIC in the target nucleus, and projectile nucleus; plot 2 (small full circles) is for the model without FZIC. The target fragmentation region is at negative Feynman- $x_{F}$, the projectile fragmentation is at positive $x_{F}$. For comparison we give also the same distribution in p-air collisions with FZIC (small empty circles). 


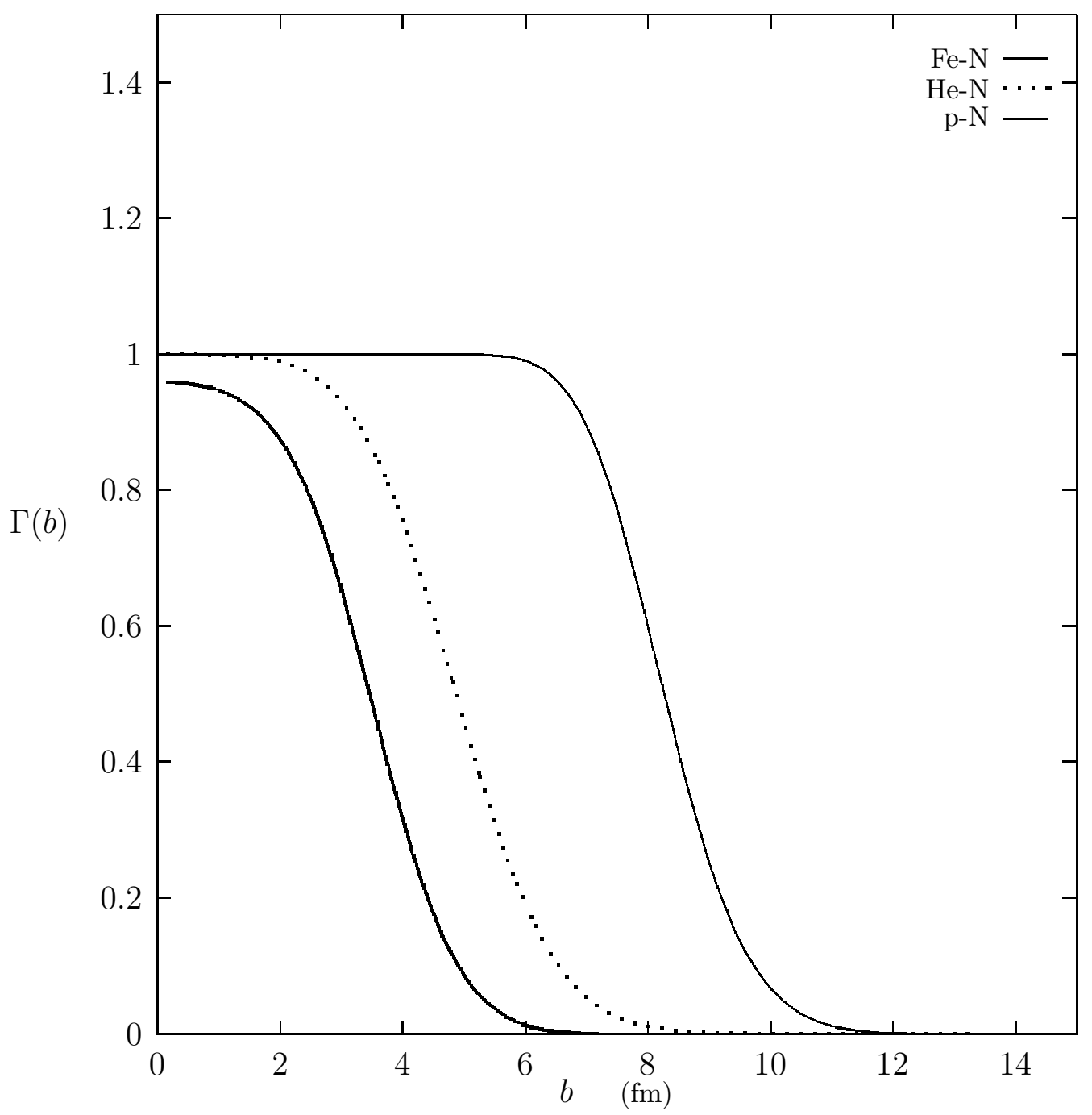

Figure 5: Glauber profile functions $\Gamma(b)$ for $\mathrm{Fe}-$ nitrogen, He-nitrogen and $\mathrm{p}-$ nitrogen collisions at $500 \mathrm{TeV}$. 